\title{
CoORdinate Systems Used in Geodesy: Basic Definitions and Concepts
}

By Tomás Soler ${ }^{1}$ and Larry D. Hothem, ${ }^{2}$ Member, ASCE

AвstRact: In order to properly apply transformations when using data derived from different space techniques, the corresponding frames should be clearly stated. Only then can a rigorous comparison of results be established. This review is an attempt to expound some of the basic definitions and concepts of reference frames to users from diverse backgrounds, who are not familiar with the geodetic terminology.

\section{INTRODUCTION}

The principal problem of geodesy may be stated as follows (Hirvonen 1960): "Find the space coordinates of any point $P$ at the physical surface $S$ of the earth when a sufficient number of geodetic operations have been carried out along $S$." Therefore, in order to know the position of $P$, the definition of an appropriate frame to which these spatial coordinates refer is of primary importance.

Due to the nature of the rotational motions of the earth and to other geodynamic phenomena, a rigorously defined, earth-fixed coordinate system at the degree of accuracy of our current observational capabilities is not presently available. Recent meetings, colloquiums, and workshops organized jointly by the International Association of Geodesy (IAG) and the International Astronomical Union (IAU) are attempts to coordinate the work of different groups in the international scientific community for the future definition and selection of reliable reference frames (e.g., see Wilkins and Mueller 1986).

In this review, only those terrestrial (earth-fixed) and local reference frames that are commonly used in geodesy will be covered.

The writers are fully aware of a proliferation of standard geodetic texts covering the topics treated here (e.g., Heiskanen and Moritz 1967; Hotine 1969; Rapp 1975; Groten 1979; Bomford 1980; Leick 1980; Torge 1980; Vaníček and Krakiwsky 1982). Nevertheless our impression is that an increasing number of users of modern space techniques-some of them not familiar with geodetic terminology - need a succinct introductory explanation of coordinate systems and their fundamental relationships (see Appendix I). These systems are constantly mentioned and are assumed to be known in the published literature. Consequently, our principal motivation was to set forth in a comprehensive concise way the most basic

'Geodesist, Natl. Geodetic Survey, Charting and Geodetic Svces., Natl. Ocean Svce., NOAA, Rockville, MD 20852; Adj. Prof., Dept. of Civ. Engrg., Virginia Polytechnic Inst. and State Univ., Northern Virginia Grad. Ctr., Falls Church, VA 22090.

${ }^{2}$ Chf., Astronomy and Space Geodesy Sect., Natl. Geodetic Survey, Charting and Geodetic Svces., Natl. Ocean Service, NOAA, Rockville, MD 20852.

Note. Discussion open until October 1, 1988. To extend the closing date one month, a written request must be filed with the ASCE Manager of Journals. The manuscript for this paper was submitted for review and possible publication on January 7, 1988. This paper is part of the Journal of Surveying Engineering, Vol. 114, No. 2, May, 1988. () ASCE, ISSN $0733-9453 / 88 / 0002-0084 / \$ 1.00+\$ .15$ per page. Paper No. 22465. 
definitions and equations, thus unifying as much as possible the nomenclature and notations that are the principal sources of confusion when different references are contrasted.

In the following sections, several important coordinate systems of geodetic significance will be presented. They are grouped into three fundamental categories: (1) Global; (2) curvilinear; and (3) local.

\section{Global Cartesian Coordinate Systems}

To standardize the notation as much as possible, all global Cartesian terrestrial coordinate systems will be represented by boldface, lowercase letters. Capital letters will be reserved for the astronomically defined inertial coordinate systems not covered in this review.

\section{$(\mathbf{x}, \mathbf{y}, \mathbf{z})$ : Conventional Terrestrial Reference Coordinate System (CTRS)} The CTRS has the following definition:

1. Origin: At the geocenter (center of mass of the earth).

2. $\mathbf{z}$-axis: Directed toward the conventional definition of the North Pole, or more precise, towards the conventional terrestrial pole (CTP) as defined by the International Earth Rotation Service (IERS).

3. $\mathbf{x}$-axis: Passes through the point of zero longitude (approximately on the Greenwich meridian) as defined by the IERS.

4. $\mathbf{y}$-axis: Forms a right-handed coordinate system with the $\mathbf{x}$ - and $\mathbf{z}$-axes.

\section{World Systems}

These are different terrestrial coordinate systems close to the CTRS, although not conventionally adopted by international agreement. These world coordinate systems are materialized by station coordinates derived from independent satellite observations and solutions accomplished by different organizations using their own software and methods.

Examples of the most widely used world systems are:

1. $(\mathbf{x}, \mathbf{y}, \mathbf{z})_{\text {WGS72 }}$ : Primarily is derived through Doppler observations and the ephemerides of the navy navigation satellite system (NNSS). Before January 4, 1987, it was also realized from pseudoranges and/or phase observations and the precise ephemerides of the navigation, satellite, timing and ranging (NAVSTAR) global positioning system (GPS).

2. $(\mathbf{x}, \mathbf{y}, \mathbf{z})_{\text {WGS84 }}$ : After January 4,1987 , was realized through pseudoranges or phase observations and the precise ephemerides of the NAVSTAR GPS. The broadcast ephemerides were switched to the WGS 84 system on January $23,1987$.

3. $(\mathbf{x}, \mathbf{y}, \mathbf{z})_{\text {SLR }}$ : Primarily is defined by satellite laser ranging (SLR) and the ephemerides of the satellite LAGEOS, e.g., Goddard Space Flight Center (GSFC) solution SL5 and University of Texas solution LSC8402. See Tapley et al. 1985 .

4. Very long baseline interferometry (VLBI) techniques, which observe extragalactic radio sources such as quasars and are strictly kinematic, can provide only orientation and scale, but not a geocentric origin. However, they can determine precisely the earth rotation parameters, ERP (i.e., polar motion components $x_{p}, y_{p}$, and Universal Time (UT1)) needed for a rigorous definition of the CTRS $(\mathbf{x}, \mathbf{y}, \mathbf{z})$. 


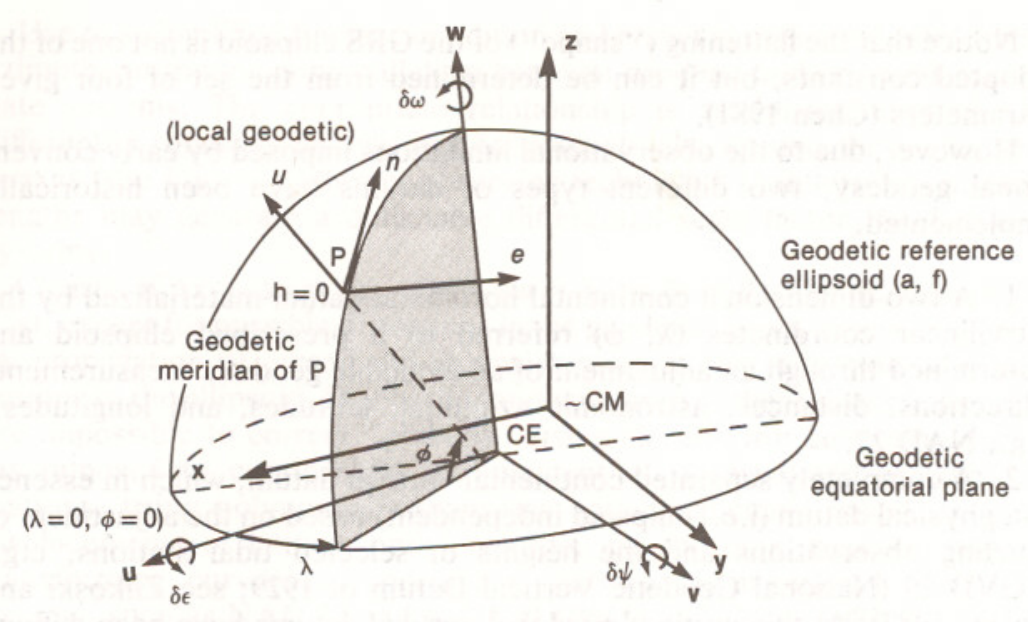

FIG. 1. Conventional Terrestrial, Geodetic, and Local Geodetic Frames

Some as yet unexplained small differences between the origins, orientation, and scale of these world coordinate systems have been found (Hothem et al. 1982). Transformation parameters between several world coordinate systems and the CTRS were recently determined by Boucher and Altamimi (1985).

\section{$(\mathbf{u}, \mathbf{v}, \mathbf{w})$ : Geodetic Coordinate Systems}

There is one geodetic coordinate system for each datum (see Fig. 1). They are defined as follows:

1. Origin: Is at the center of the reference ellipsoid used for defining the datum in question.

2. w-axis: Coincides with the semiminor axis $b$ of the reference ellipsoid.

3. u-axis: Passes through the point $\lambda=0, \phi=0$. (See later in the corresponding section, the definition of curvilinear geodetic coordinates).

4. v-axis: Forms a right-handed triad with the $\mathbf{u}$ - and $\mathbf{w}$-axes.

Examples of Cartesian geodetic coordinate systems derived after transforming the datum curvilinear coordinates (including heights) into Cartesian coordinates are: (1) (u, v, w) $)_{\text {NAD27 }} ;(2)(\mathbf{u}, \mathbf{v}, \mathbf{w})_{\text {NAD83 }} ;$ and (3) (u, v, w) European datum .

A datum (strictly speaking a reference surface) is often based on the best-fitting ellipsoid to the earth or any of its regions. Consequently, two wide categories of datums should be mentioned: (1) Global or absolute (geocentric); and (2) regional or continental (nongeocentric).

The "ideal" global datum is defined by the earth's best-fitting ("mean earth") ellipsoid. Because the earth is rotating and has mass, its best physical approximation is given through the four parameters of a geodetic reference system (GRS), namely: (1) $a$, equatorial radius; (2) $G M$, geocentric gravitational constant; (3) $J_{2}$, dynamical form factor; and (4) $\omega$, earth's angular velocity. 
Notice that the flattening ("shape") of the GRS ellipsoid is not one of the adopted constants, but it can be determined from the set of four given parameters (Chen 1981).

However, due to the observational limitations imposed by early conventional geodesy, two different types of datums have been historically implemented:

1. A two-dimensional continental horizontal datum materialized by the curvilinear coordinates $(\lambda, \phi)$ referred to a prescribed ellipsoid and determined through an adjustment of all available geodetic measurements (directions, distances, astronomic azimuths, latitudes, and longitudes), e.g., NAD 27.

2. A completely separated continental vertical datum, which in essence is a physical datum (i.e., ellipsoid independent) based on the adjustment of leveling observations and the heights of selected tidal stations, e.g., NGVD 29 (National Geodetic Vertical Datum of 1929; see Zilkoski and Balazs 1987). At this writing, no global vertical datums have been defined or adopted.

The recently completed NAD 83 (see Wade 1986; Bossler 1987) is an example of a global horizontal datum referred to the ellipsoid defined by the parameters of the GRS 80 adopted at the XVII General Assembly of the IUGG (International Union of Geodesy and Geophysics) held in Canberra, Australia, in December 1979 (Moritz 1980, 1984; Chovitz 1981; Burkholder 1984). Although the NAD 83 incorporates observations determined through modern space techniques, such as Doppler and VLBI, nevertheless the adjustment does not include as unknowns the geodetic height of the points. Consequently, "the NAD 83 remains a horizontal datum.

With Doppler or GPS receivers, Cartesian coordinates $(\mathbf{x}, \mathbf{y}, \mathbf{z})_{\mathrm{WGS} 72}$ were initially determined from the raw observations. These coordinates can be transformed into curvilinear $(\lambda, \phi, h)_{\text {WGS72 }}$ using the semimajor axis and flattening of the so-called WGS 72 ellipsoid. In this instance, therefore, we may talk about curvilinear coordinates in the "WGS 72 datum." Incidentally, this WGS 72 datum, although global (i.e., the reference ellipsoid is assumed geocentric), is not based on any internationally adopted GRS. After January 4 and 23, 1987, the precise and broadcast ephemerides of the GPS satellite constellation were both changed to the (x, $\mathbf{y}, \mathbf{z})_{\text {WGS84 }}$ system. As mentioned earlier, an ellipsoid must be selected to transform Cartesian to curvilinear $(\lambda, \phi, h)_{\mathrm{WGS} 4}$ coordinates. Accordingly, we may now talk about the satellite-determined "WGS 84 datum." The suggested ellipsoid for WGS 84 was the GRS 80 ellipsoid. However, there is an insignificant difference between the flattenings of the two ellipsoids, which does not affect practical results (DMA 1987).

Continental horizontal geodetic datums established a long time ago using classical geodetic observations and procedures are nongeocentric. Observed astronomic latitude and longitude values, the adopted geoidal undulation and deflections of the vertical at the datum origin (e.g., station MEADES RANCH in Kansas, for the NAD 27), and the selected parameters of the fitting ellipsoid, all influence the shifts $\Delta \mathbf{x}, \Delta \mathbf{y}, \Delta \mathbf{z}$ of the origin of the $(\mathbf{u}, \mathbf{v}, \mathbf{w})$ Cartesian geodetic system with respect to the geocenter. 
Use of a simplified Laplace condition and errors in measured astronomic azimuths introduce nonparallelism between geodetic and/or world coordinate systems. The appropriate relationship is established through the differential rotations $\delta \varepsilon, \delta \psi$, and $\delta \omega$ respectively, around the $\mathbf{u}-, \mathbf{v}-$, and w-axes (see Fig. 1). Different observing techniques to determine baseline lengths may generate a detectable differential scale factor, $\delta s$, between systems.

Clearly, if $\Delta \mathbf{x}=\Delta \mathbf{y}=\Delta \mathbf{z}=\delta \varepsilon=\delta \psi=\delta \omega=\delta s=0$, then $(\mathbf{u}, \mathbf{v}, \mathbf{w})=(\mathbf{x}$ $\mathbf{y}, \mathbf{z})$, except for possible distortions in the geodetic datum generally caused by propagation of errors and inconsistencies in the reduction of observations to the ellipsoid. Due to the random nature of these distortions, they are impossible to correct "globally" using closed-form equations. Local (as opposed to continental) readjustment of observations or transformations, using polynomial expansions with coefficients based on local displacements of latitude and longitude, are the most rigorous way of transforming curvilinear coordinates between conventional horizontal datums, such as NAD 27 and NAD 83 (Wade and Doyle 1987).

\section{Curvilinear Coordinate Systems}

Due to the nature of the basic reference surface used in geodetic problems, sometimes it is convenient to employ curvilinear coordinates instead of spatial rectangular coordinates. This is especially true when an ellipsoid is adopted as reference.

\section{$(\lambda, \phi, h)$ : Curvilinear Geodetic Coordinates}

These coordinates are defined as follows (see Figs. 1 and 2):

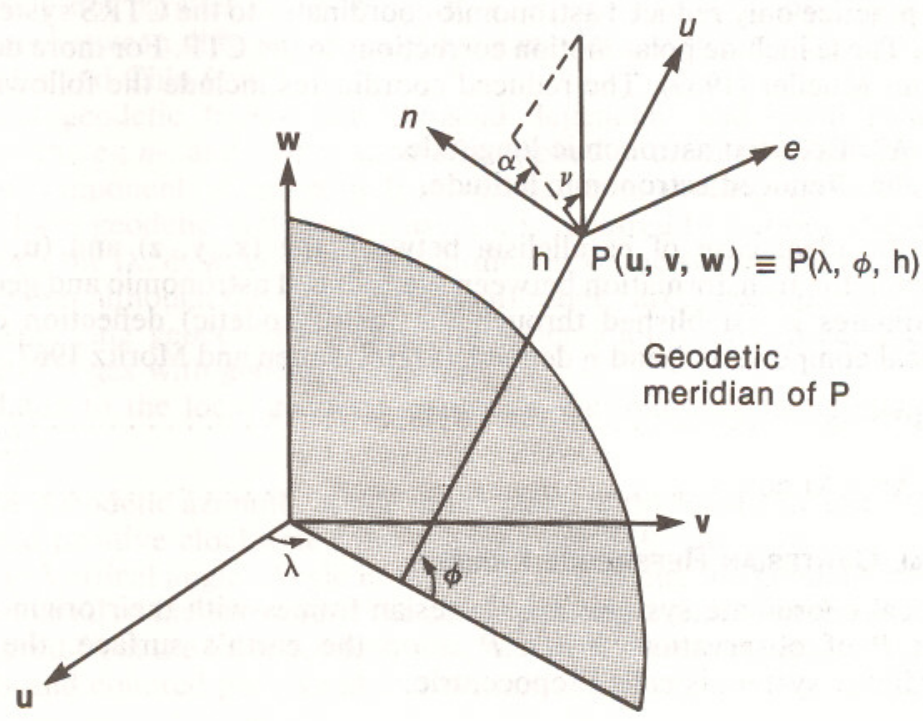

FIG. 2. Geodetic and Local Geodetic Frames 
1. $\lambda$ : Geodetic longitude. Angle between the plane uw and the geodetic meridian plane of point $P$ measured positive toward the east (or west for the NAD 27 and NAD 83 datums); $0 \leq \lambda \leq 2 \pi$.

2. $\phi$ : Geodetic latitude. Angle between the normal to the ellipsoid at $P$ and the plane uv; $-\pi / 2 \leq \phi \leq \pi / 2$.

3. $h$ : Geodetic height. Distance along the normal to the reference ellipsoid between $P$ and the surface of this ellipsoid. Some authors use the term ellipsoidal height for this parameter.

Note that a point $P$ may have different sets of curvilinear geodetic coordinates, each one based on independent geodetic datums (i.e., nongeocentric NAD 27 in contrast to geocentric NAD 83).

\section{$(\Lambda, \Phi, H)$ : Natural (or Astronomic) Coordinates}

Natural coordinates are strictly based on physical properties and are unique at each point $P$. They include the following:

1. $\Lambda$ and $\Phi$ : Astronomic longitude and latitude. These curvilinear coordinates are directly determined from observations; therefore, they refer to the instantaneous earth rotation axis and equator. The normal to the ellipsoid is replaced by the direction of the plumb line.

2. $H$ : Orthometric height. Also loosely referred to as elevation or mean sea level height. It is derived from leveling observations and gravity data; it is the distance along the plumb line from $P$ to the geoid. This third natural coordinate may be replaced by the value of the geopotential at $P$. The geoid is the equipotential surface of the earth gravity field that best approximates mean sea level.

An important formula relates $h$ and $H$ with $\mathbf{N}_{g}$ (undulation or geoidal height): $h \simeq H+\mathbf{N}_{g}$.

In practice only reduced astronomic coordinates to the CTRS system are used. These include polar motion corrections to the CTP. For more details, consult Mueller (1969). The reduced coordinates include the following:

1. $\Lambda^{*}$ : Reduced astronomic longitude.

2. $\Phi^{*}:$ Reduced astronomic latitude.

In the ideal case of parallelism between the $(\mathbf{x}, \mathbf{y}, \mathbf{z})$ and $(\mathbf{u}, \mathbf{v}, \mathbf{w})$ systems, the transformation between the reduced astronomic and geodetic coordinates is established through the (astrogeodetic) deflection of the vertical components $\xi$ and $\eta$ defined as (Heiskanen and Moritz 1967, p. 83)

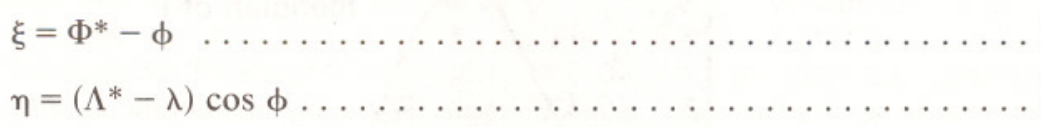

\section{Local Cartesian Reference Frames}

Local coordinate systems are Cartesian frames with their origin at the point $P$ of observation. When $P$ is on the earth's surface, the local coordinate system is called topocentric.

$(x, y, z)$ : Local (Terrestrial) Frames

Local terrestrial frames are defined as follows: 
1. Origin: Any point $P(x, y, z)$.

2. The $x-, y-$, and $z$-axes are parallel to any prescribed terrestrial $(\mathbf{x}, \mathbf{y}$, z) frame.

We may define a local terrestrial frame at any point with respect to any of the world systems mentioned before, e.g., $(x, y, z)_{\text {WGS72 }}$ or $(x, y$, z) WGS84.

These types of frames are used to report results from relative positioning techniques (e.g., GPS relative satellite positioning or VLBI). Because the relative coordinates of a point $B$ with respect to another point $A$ (assumed known) are determined, sometimes the notations $(d x, d y, d z)$ or $(\Delta x, \Delta y$, $\Delta z$ ) are used, where $d x \equiv \Delta x=\mathbf{x}_{B}-\mathbf{x}_{A}$, etc. This implies that the coordinates of the forepoint (remote station) $B$ with respect to the standpoint (reference station) $A$ are known in a local frame at $A$, which is parallel to any terrestrial (world) system (e.g., WGS 84).

\section{$(e, n, u)$ : Local Geodetic Frame}

Local geodetic frames (see Figs. 1 and 2) are defined as follows:

1. Origin: Any point $P(\lambda, \phi, h)$ referred to a given ellipsoid.

2. $u$-axis: Normal through $P$ to the reference ellipsoid. The positive sign in the outward (geodetic zenith) or "up" direction.

3. $e$-axis: Normal to $u$ and to the geodetic meridian plane (when $h=0$, tangent to the geodetic parallel of $P$ ). Positive (east) in the direction of increasing $\lambda$.

4. $n$-axis: Perpendicular to $e$ and $u$ forming a right-handed triad (when $h$ $=0$, tangent to the geodetic meridian of $P$ ). Positive (north) in the direction of increasing $\phi$.

Notice that the local geodetic frame $(e, n, u)$ is right-handed but is generally not parallel to the frame $(\mathbf{u}, \mathbf{v}, \mathbf{w})$ previously defined. Transformations between different types of local geodetic systems were discussed by Soler and Chin (1985).

Local geodetic frames are ellipsoid dependent and small rotations around the $e_{-}, n-$, and $u$-axes should be applied in order to transform local vector components at any point from one datum to another. Consequently, when local geodetic systems are used, it is required to mention the datum to which the $(\lambda, \phi, h)$ curvilinear coordinates of the standpoint refer. Also notice that although the local (terrestrial) frames are always parallel to the corresponding geocentric system, the orientation of the local geodetic frame changes with geodetic position.

Related to the local geodetic frame are the following parameters (see Fig. 2):

1. $\alpha$ : Geodetic azimuth. Angle in the plane of the local geodetic horizon counted positive clockwise from geodetic north; $0 \leq \alpha \leq 2 \pi$.

2. $v$ : Vertical angle. Angle in the plane containing the geodetic vertical (i.e., normal to the ellipsoid of the corresponding datum) and the forepoint measured from the local geodetic horizon to the direction between the two points and counted positive above the horizon; $-\pi / 2 \leq \nu \leq \pi / 2$.

\section{$\left(e^{*}, n^{*}, u^{*}\right)$ : Local Astronomic Frame}

Local astronomic frames are defined as follows: 
1. Origin: At point $P$.

2. $u^{*}$-axis: Normal through $P$ to the geopotential surface of $P$ (i.e., tangent at $P$ to the plumb line passing through $P$ ). Positive outwards (i.e., astronomic zenith).

3. $e^{*}$-axis: Normal to $u^{*}$ and to the CTP referred astronomical meridian of $P$. Positive east in the direction of increasing astronomic longitude.

4. $n^{*}$-axis: Normal to $u^{*}$ and $e^{*}$ forming a right-handed system. Positive north in the direction of increasing astronomic latitude.

The relationship between the astronomic and geodetic azimuth and vertical angle is obtained from Heiskanen and Moritz (1967, pp. 186 and 190):

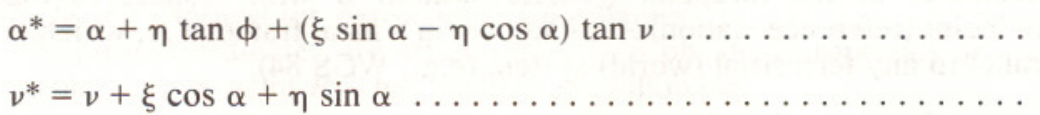

where $\eta$ and $\xi$ are the deflection of the vertical components defined previously. Incidentally, the so-called Laplace's equation (or condition) is merely $\Delta \alpha=\alpha^{*}-\alpha$.

\section{Conclusions}

When using relative positioning methods, it is general practice to express the data in local frames with origin at the fixed station. That type of selection obviously will establish the required relationship of the location of any point with respect to the one assumed known.

The question that may be asked now is which of all the local frames previously discussed is optimum or most appropriate for representing the components of each individual baseline vector. In the writers' opinion, available baseline components in various data bases should always be given in a clearly specified local frame of the terrestrial type (e.g., the local CTRS, WGS 72 or WGS 84 systems at the fixed point). The analyst retrieving the data should then decide which other local frame is the most appropriate to express the final results. This naturally will depend on the particular problem under study (e.g., geodetic surveying, dynamic positioning, or geodynamics).

Local terrestrial frames (i.e., frames located at the observation point and parallel to any previously defined terrestrial frame) are difficult to visualize. Even a trained observer located on the surface of the earth will have a hard time knowing, for example, the direction of an axis parallel to the Greenwich meridian from the observation point. To remedy this problem, a local geodetic frame may be used to express vector components in which the three axes will point, respectively, to the geodetic east, north, and zenith. This selection should always be exercised when treating the short baselines typically used in surveying practice.

The importance of local geodetic frames is crucial when the final statistics of the GPS solutions are portrayed. Although the components of the baseline vector in the local geodetic frame are not very meaningful for long baselines, nevertheless, the standard or root mean square (rms) errors of the determined position in this particular frame are very useful for interpreting the results. Notice that a check of the errors in longitude, 
latitude, and geodetic height will provide better insight into problems such as dependence of the solution on orbital geometry, ionospheric and tropospheric delays, timing errors, etc. "Clearly, these types of analyses will be very difficult to interpret if the final standard errors are expressed only in the Cartesian global or local terrestrial frames.

\section{Appendix I. Important Equations Used in Geodetic TRANSFORMATIONS}

All the equations described in Appendix I are based on the following conventions:

1. All coordinate systems are right-handed.

2. Positive rotations are counterclockwise rotations as viewed looking towards the origin of the coordinate system.

3 . The geodetic longitude $\lambda$ is counted positive toward the east.

Transformation from Curvilinear Geodetic $(\lambda, \phi, h)$ to Cartesian Geodetic (u, v, w) Coordinates

$$
\left\{\begin{array}{l}
\mathbf{u} \\
\mathbf{v} \\
\mathbf{w}
\end{array}\right\}=\left\{\begin{array}{c}
(N+h) \cos \phi \cos \lambda \\
(N+h) \cos \phi \sin \lambda \\
{\left[N\left(1-e^{2}\right)+h\right] \sin \phi}
\end{array}\right\} \text {. }
$$

where $(\lambda, \phi, h)$ are given in any arbitrary datum based on an ellipsoid of semimajor axis $a$ and flattening $f$, and

$N=\frac{a}{W}$ principal radius of curvature in the prime vertical plane ..

$$
W=\left(1-e^{2} \sin ^{2} \phi\right)^{\frac{1}{2}}
$$

$e^{2}=2 f-f^{2}$

Transformation from Cartesian Geodetic $(\mathrm{u}, \mathbf{v}, \mathbf{w})$ to Curvilinear $(\lambda, \phi, h)$ Coordinates

The following noniterative (and for all practical applications, rigorous) method is based on the work of Bowring (1985):

$\tan \lambda=\frac{\mathbf{v}}{\mathbf{u}}$

$\tan \phi=\frac{\left(\mathbf{w}+e^{2} a \sin ^{3} \mu\right) l(1-f)}{\left(p-e^{2} a \cos ^{3} \mu\right)}$

$h=p \cos \phi+\mathbf{w} \sin \phi-\left(\frac{a^{2}}{N}\right)$

where $p=\left(\mathbf{u}^{2}+\mathbf{v}^{2}\right)^{\frac{1}{2}}$

$r=\left(p^{2}+\mathbf{w}^{2}\right)^{\frac{1}{2}}$ 
$\tan \mu=\frac{\mathbf{w}(1-f)}{p}\left[1+\frac{e^{2} a}{r(1-f)}\right]$.

Seven-Parameter (Similarity) Transformation between Any Two

Cartesian Systems, e.g., from $(u, v, w)$ to $(x, y, z)$

$\left\{\begin{array}{l}\mathbf{x} \\ \mathbf{y} \\ \mathbf{z}\end{array}\right\}=\left\{\begin{array}{l}\Delta \mathbf{x} \\ \Delta \mathbf{y} \\ \Delta \mathbf{z}\end{array}\right\}+(1+\delta s)\left[\begin{array}{ccc}1 & \delta \omega & -\delta \psi \\ -\delta \omega & 1 & \delta \varepsilon \\ \delta \psi & -\delta \varepsilon & 1\end{array}\right]\left\{\begin{array}{l}\mathbf{u} \\ \mathbf{v} \\ \mathbf{w}\end{array}\right\}$

where $\Delta \mathbf{x}, \Delta \mathbf{y}, \Delta \mathbf{z}=$ coordinates of the origin of the frame $(\mathbf{u}, \mathbf{v}, \mathbf{w})$ in the frame $(\mathbf{x}, \mathbf{y}, \mathbf{z}) ; \delta \varepsilon, \delta \psi, \delta \omega=$ differential rotations, respectively, around the axes $(\mathbf{u}, \mathbf{v}, \mathbf{w})$ to establish parallelism with the $(\mathbf{x}, \mathbf{y}, \mathbf{z})$ frame; and $\delta s=$ differential scale change.

Transformation from Local $(x, y, z)$ to Local Geodetic $(e, n, u)$

Coordinates

$\left\{\begin{array}{l}e \\ n \\ u\end{array}\right\}=[\mathbf{R}]\left\{\begin{array}{l}x \\ y \\ z\end{array}\right\}$

where the rotation matrix $[\mathbf{R}]$ may be computed from

$[\mathbf{R}]=R_{1}\left(\frac{1}{2} \pi-\phi\right) R_{3}\left(\lambda+\frac{1}{2} \pi\right)$
$=\left[\begin{array}{ccc}-\sin \lambda & \cos \lambda & 0 \\ -\sin \phi \cos \lambda & -\sin \phi \sin \lambda & \cos \phi \\ \cos \phi \cos \lambda & \cos \phi \sin \lambda & \sin \phi\end{array}\right]$

The geodetic azimuth and vertical angle of the forepoint with respect to the standpoint can be computed, respectively, by

$\tan \alpha=\frac{e}{n}$

$\tan v=\frac{u}{\left(e^{2}+n^{2}\right)^{\frac{1}{2}}}$

General Differential Transformation of Curvilinear Geodetic

Coordinates from Datum D1 to Datum D2 .

The final geodetic coordinates on datum D2 are

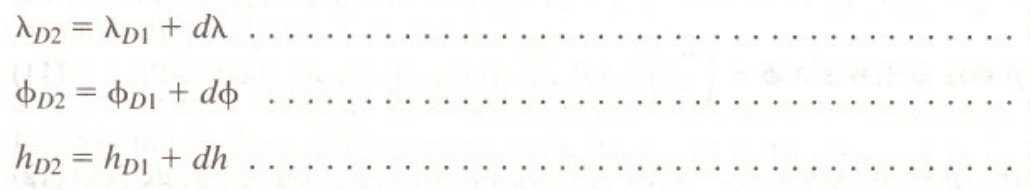

The differential corrections $(d \lambda, d \phi, d h)$ may be obtained from the matrix equation 


$$
\left\{\begin{array}{c}
(N+h) \cos \phi d \lambda \\
(M+h) d \phi \\
d h
\end{array}\right\}=[\mathbf{R}]\left\{\left\{\begin{array}{l}
d \mathbf{u} \\
d \mathbf{v} \\
d \mathbf{w}
\end{array}\right\}_{7 \text { parameters }} \quad+\left\{\begin{array}{l}
d \mathbf{u} \\
d \mathbf{v} \\
d \mathbf{w}
\end{array}\right\}_{\delta a, \delta f}\right\} \ldots
$$

where $N$ and [R] were given previously by Eqs. 6 and 17, respectively, and $\left\{\begin{array}{l}d \mathbf{u} \\ d \mathbf{v} \\ d \mathbf{w}\end{array}\right\}_{7 \text { par }}=\left\{\begin{array}{l}\Delta \mathbf{x} \\ \Delta \mathbf{y} \\ \Delta \mathbf{z}\end{array}\right\}+\left[\begin{array}{ccc}\delta s & \delta \omega & -\delta \psi \\ -\delta \omega & \delta s & \delta \varepsilon \\ \delta \psi & -\delta \varepsilon & \delta s\end{array}\right]\left\{\begin{array}{c}\mathbf{u} \\ \mathbf{v} \\ \mathbf{w}\end{array}\right\}$

$\left\{\begin{array}{l}d \mathbf{u} \\ d \mathbf{v} \\ d \mathbf{w}\end{array}\right\}_{\delta a, \delta f}=-[\mathbf{D}]\left\{\begin{array}{c}\delta a \\ \delta f\end{array}\right\}$

$[\mathbf{D}]=\left[\begin{array}{cc}\cos \phi \cos \lambda / W & a(1-f) \sin ^{2} \phi \cos \phi \cos \lambda / W^{3} \\ \cos \phi \sin \lambda W & a(1-f) \sin ^{2} \phi \cos \phi \sin \lambda W^{3} \\ \left(1-e^{2}\right) \sin \phi / W & \left(M \sin ^{2} \phi-2 N\right)(1-f) \sin \phi\end{array}\right]_{D \mid}$

The differential parameters $\Delta \mathbf{x}, \Delta \mathbf{y}, \Delta \mathbf{z} ; \delta \varepsilon, \delta \psi, \delta \omega ;$ and $\delta s$ were defined previously and

$\delta \bar{a}=a_{D 2}-a_{D 1}$

$\delta f=f_{D 2}-f_{D 1}$

$\delta a=\delta \bar{a}+a_{D 1} \delta s$

$\delta a$ is the total change in reference ellipsoid semimajor axis when a differential scale change $\delta s$ is also involved in the transformation between datums. See Soler and van Gelder (1987). Finally

$M=\frac{a\left(1-e^{2}\right)}{W^{3}}$.

which is the principal radius of curvature in the plane of the meridian.

The exact correspondence of the expression shown in Eq. 23 with the often-quoted Molodenskii equations (Molodenskii et al. 1960) may be consulted in Soler (1976).

Transformation between Local Geodetic $(e, n, u)$ and Local Astronomic $\left(e^{*}, n^{*}, u^{*}\right)$ Frames

$$
\left\{\begin{array}{l}
e^{*} \\
n^{*} \\
u^{*}
\end{array}\right\} \simeq\left[\begin{array}{ccc}
1 & \eta \tan \phi & -\eta \\
-\eta \tan \phi & 1 & -\xi \\
\eta & \xi & 1
\end{array}\right]\left\{\begin{array}{l}
e \\
n \\
u
\end{array}\right\}
$$

where $\phi=$ the geodetic latitude of the point; and $\eta$ and $\xi$ are, respectively, the components of the deflection of the vertical along the prime vertical (positive east) and meridian (positive north). 
Appendix II. Important Parameters Used in Geodetic TRANSFORMATIONS

Tables 1 and 2 show the adopted transformation parameters for several world systems to be used in the equations described in Appendix I.

TABLE 1. Adopted Transformation Parameters for Several World Systems

\begin{tabular}{l|c|c|c|c|c}
\hline \hline $\begin{array}{c}\text { Coordinate system } \\
\text { (datum) } \\
(1)\end{array}$ & $\begin{array}{c}\Delta \mathbf{x}=\Delta \mathbf{y} \\
(\mathrm{m}) \\
(2)\end{array}$ & $\begin{array}{c}\Delta \mathbf{z} \\
(\mathrm{m}) \\
(3)\end{array}$ & $\begin{array}{c}\delta \varepsilon=\delta \psi \\
(\operatorname{arcsec}) \\
(4)\end{array}$ & $\begin{array}{c}\delta \omega \\
(\operatorname{arcsec}) \\
(5)\end{array}$ & $\begin{array}{c}\delta s \\
(\mathrm{ppm}) \\
(6)\end{array}$ \\
\hline $\begin{array}{l}\text { NWL 9D (or NSWC } \\
\text { 9Z2) to WGS 72 }\end{array}$ & 0 & 0 & 0 & -0.26 & -0.827 \\
$\begin{array}{l}\text { NWL 9D to WGS 84 (or } \\
\text { NAD 83) }\end{array}$ & 0 & $+4.5^{\mathrm{a}}$ & 0 & $-0.814^{\mathrm{b}}$ & $-0.6^{\mathrm{b}}$ \\
$\begin{array}{l}\text { WGS 72 to WGS 84 (or } \\
\text { NAD 83) }\end{array}$ & 0 & $+4.5^{\mathrm{a}}$ & 0 & -0.554 & +0.227 \\
\hline \\
a Determined from comparison with SLR. \\
bDetermined from comparison with VLBI. \\
\hline
\end{tabular}

TABLE 2. Parameters of Some Adopted Ellipsoids

\begin{tabular}{c|c|c|c}
\hline \hline $\begin{array}{c}\text { Coordinate system } \\
\text { (datur:; } \\
\text { (1) }\end{array}$ & $\begin{array}{c}\text { Reference } \\
\text { ellipsoid } \\
\text { used } \\
(2)\end{array}$ & $\begin{array}{c}a \\
(\mathrm{~m})\end{array}$ & $\begin{array}{c}1 / f \\
(3)\end{array}$ \\
\hline NAD 27 & Clarke 1866 & 6378206.4 & 298.9786982 \\
NWL 9D = NSWC 9Z2 & WGS 66 & 6378145.0 & 298.25 \\
WGS 72 & WGS 72 & 6378135.0 & 298.26 \\
NAD 83 & GRS 80 & 6378137.0 & 298.257222101 \\
WGS 84 & WGS 84 & 6378137.0 & 298.257223563 \\
\hline
\end{tabular}

Note: GRS = Geodetic Reference System; NAD = North American Datum; NSWC $=$ Nava Surface Warfare Center; NWL = Naval Weapons Laboratory; WGS $=$ World Geodetic System; $a=$ semimajor axis (size); $f=$ flattening (shape).

\section{Appendix III. References}

Bomford, G. (1980). Geodesy, 4th Ed., Clarendon Press, Oxford, England.

Bossler, J. D. (1987). "Geodesy solves 900,000 equations simultaneously." Eos, Trans. AGU, American Geophysical Union, 68, 569.

Boucher, C., and Altamimi, Z. (1985). "Towards an improved realization of the BIH terrestrial frame." Proc. Int. Conf. Earth Rotation and Terrestrial Reference Frame, Dept. of Geodetic Sci. and Surveying, Ohio State Univ., Columbus, Ohio, pp. 551-564.

Bowring, B. R. (1985). "The accuracy of geodetic latitude and height equations." Survey Review, 28, 202-206.

Burkholder, E. F. (1984). "Geometrical parameters of the geodetic reference system 1980." Surveying and Mapping, 44, 339-340.

Chen, J. Y. (1981). "Formulas for computing ellipsoidal parameters." Bulletin Géodésique, 55, 170-178.

Chovitz, B. H. (1981). "Modern geodetic earth reference models." Eos, Trans. $A G U$, American Geophysical Union, 62, 65-67. 
Defense Mapping Agency (1987). "Department of Defense World Geodetic System 1984 -Its definition and relationships with local geodetic systems." DMA Technical Report 8350.2, Defense Mapping Agency, Washington, D.C.

Groten, E. (1979). Geodesy and the earth's gravity field. I and II, F. Dümmler, Bonn, Federal Republic of Germany.

Heiskanen, W. A., and Moritz, H. (1967). Physical Geodesy. W. H. Freeman and Co., San Francisco, Calif.

Hirvonen, R. A. (1960). "New theory of the gravimetric geodesy." Annales Academiae Scientiarum Fennicae, Series A, III, Geologica-Geographica, 56, Helsinki, Finland.

Hothem, L. D., Vincenty, T., and Moose, R. E. (1982). "Relationship between Doppler and other advanced geodetic system measurements based on global data." Proc. 3rd Int. Geodetic Symp. on Satellite Doppler Positioning, Defense Mapping Agency, Washington, D.C.

Hotine, M. (1969). Mathematical geodesy. ESSA Monograph No. 2, U.S. Dept. of Commerce, Natl. Oceanic and Atmospheric Admin., Rockville, Md.

Leick, A. (1980). "Geometric geodesy/3D geodesy/Conformal mapping." Lecture notes, published by Dept. of Civ. Engrg., Univ. of Maine at Orono, Orono, Maine.

Molodenskii, M. S., Eremeev, V. F., and Yurkina, M. I. (1960). Methods for study of the external gravitational field and figure of the earth. Natl. Tech. Info. Svce., Springfield, Va.

Moritz, H. (1980). "Geodetic reference system 1980." Bulletin Géodésique, 54, 395-405.

Moritz, H. (1984). "Geodetic reference system 1980." Bulletin Géodésique, 58, 388-398.

Mueller, I. I. (1969). Spherical and practical astronomy as applied to geodesy. Frederik Ungar Publ. Co., New York, N.Y.

Rapp, R. H. (1975). "Geometric geodesy, Vols. I \& II." Lecture notes, published by Dept. of Geodetic Sci. and Surveying, Ohio State Univ., Columbus, Ohio.

Soler, T. (1976). "On differential transformations between Cartesian and curvilinear (geodetic) coordinates." Report No. 236, Dept. of Geodetic Sci., Ohio State Univ., Columbus, Ohio.

Soler, T., and Chin, M. (1985). "On transformation of covariance matrices between local Cartesian coordinate systems and commutative diagrams." Tech. Papers, 45th Annual Meeting, American Congress of Surveying and Mapping, Washington, D.C., 393-406.

Soler, T., and van Gelder, B. H. W. (1987). "On differential scale changes and the satellite Doppler system z-shift." Geophysical Journal Royal Astronomical Society, 91, 639-656.

Tapley, B. D., Schutz, B. E., and Eanes, R. J. (1985). "Station coordinates, baselines, and Earth rotation from LAGEOS laser ranging: 1976-1984." $J$. Geophys. Res., 90, 9235-9248.

Torge, W. (1980). Geodesy. Walter de Gruyter, New York, N.Y.

Vanícek, P., and Krakiwsky, E. (1982). Geodesy: The concepts. North-Holland Publishing Co., New York, N.Y.

Wade, E. B. (1986). "Impact of North American datum of 1983." J. Surv. Engrg., ASCE, 112, 49-62.

Wade, E. B., and Doyle, D. R. (1987). "Datum transformation from NAD 27 to NAD 83." Tech. Papers, 47th Annual Meeting, American Congress of Surveying and Mapping, Washington, D.C., 27-36.

Wilkins, G. A., and Mueller, I. I. (1986). "Rotation of the earth and the terrestrial reference system." Eos, Trans. AGU, American Geophysical Union, 67, 601, and 604-605.

Zilkoski, D. B., and Balazs, E. I. (1987). "Status of NAVD 88 datum definition." Techn. Papers, 47th Annual Meeting, American Congress of Surveying and Mapping, Washington, D.C., 66-78. 


\section{Appendix IV. Notation}

The following symbols are used in this paper:

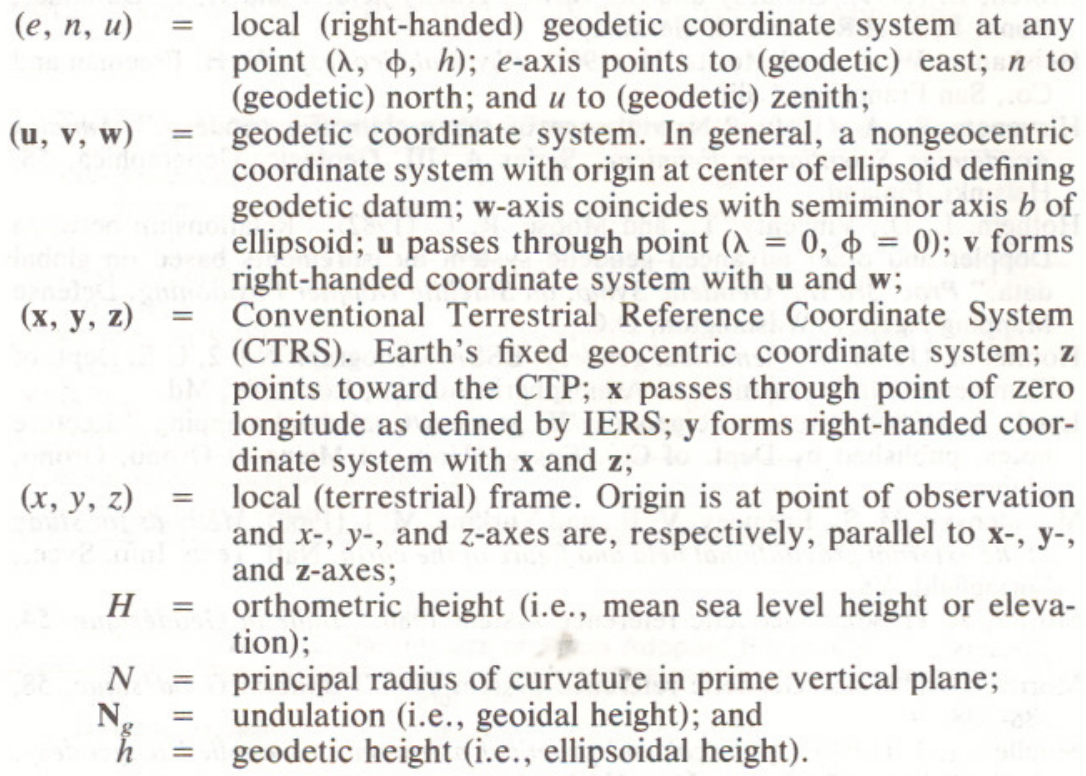

